\title{
A Vulnerabilidade dos Consumidores Obesos na Perspectiva da Pesquisa Transformativa do Consumidor
}

\section{The Vulnerability of Obese Consumers from the Perspective of Consumer Transformative Research}

\author{
Bruna Lourena de Lima Dantas ${ }^{1}$ \\ Thiago Assunção de Moraes ${ }^{2}$ \\ Hellen Taynan da Silva Cavalcanti ${ }^{3}$ \\ Janayna Souto Leal ${ }^{4}$ \\ Bianca Gabriely Ferreira Silva ${ }^{5}$
}

\begin{abstract}
Resumo
A busca por praticidade na alimentação aliada ao ambiente obesogênico construído pela indústria alimentícia causa desequilíbrios nos sistemas de marketing e pode acarretar problemas de obesidade em parcela significativa da população. Consequentemente, prejudicam a saúde física e psicológica individual, evoluindo para transtornos mais graves e tornando-se um problema de saúde pública. Nas relações de consumo, os efeitos da ingestão de produtos industrializados ainda são desconhecidos por boa parte dos consumidores e a falta de conhecimento ou controle pelos consumidores provoca a sua vulnerabilidade, desequilibrando o sistema de marketing. Essa condição de vulnerabilidade é potencializada pela omissão ou falsemaneto da composição dos alimentos nas embalagens, por exemplo, aliada a outras externalidades dos sistemas de marketing. A partir disso, a pesquisa transformativa do consumidor apresenta soluções, como a educação do consumidor, para a minimização de suas vulnerabilidades. O consumidor obeso é vulnerável, pois nem sempre tem conhecimento ou controle sobre os alimentos que consome, além de possuir características biofísicas que podem gerar hostilidade nas relações de consumo. Nesse contexto, o objetivo do presente artigo é discutir como se dá a vulnerabilidade dos consumidores obesos ou com sobrepeso a partir do consumo alimentar. Além de contribuir para uma visão interdisciplinar da obesidade, o aritog apresenta uma nova lente de análise, a perspectiva da pesquisa transformativa do consumidor, para visualização de um problema que, ao mesmo tempo que é individual, tem impactos sociais, políticos e econômicos.
\end{abstract}

Palavras-chave: Comportamento do Consumidor. Vulnerabilidade. Obesidade. Artigo Teórico.

\section{Abstract}

The search for practicality in food combined with the obesogenic environment built by the food industry causes imbalances in marketing systems and can lead to obesity problems in a significant portion of the population. Consequently, they harm individual physical and psychological health, evolving into more serious disorders and becoming a public health problem. In consumer relations, the effects of ingesting industrialized products are still unknown by most consumers and the lack of knowledge or control by consumers causes their vulnerability, unbalancing the marketing system. This condition of vulnerability is enhanced by the omission or misrepresentation of the composition of food on the packaging, for example, combined with other externalities of marketing systems. From this, transformative consumer research presents solutions, such as consumer education to minimize their

Doutora e Mestre em Administração pela Universidade Federal da Paraiba (UFPB). Professora Substituta da Universidade Federal Rural do SemiÁrido (UFERSA). Doutor e Mestre em Administração pela UFPB. Professor Adjunto da Universidade Estadual do Piaú (UESPI). 
vulnerabilities. Obese consumers are vulnerable, as they do not always have knowledge or control over the foods they consume, in addition to having biophysical characteristics that can generate hostility in consumer relations. In this context, this article aims to discuss how the vulnerability of obese or overweight consumers occurs due to food consumption. In addition to contributing to an interdisciplinary view of obesity, the article presents a new analytical lens, the perspective of transformative consumer research, to visualize a problem that, while being individual, has social, political, and economic impacts.

Keywords: Consumer Behavior. Vulnerability. Obesity. Theoretical Article.

\section{INTRODUÇÃO}

A obesidade já se estabelece como uma crise de saúde global e um fator de impacto de mortalidade. O aumento da obesidade e do sobrepeso, inclusive em populações de países em desenvolvimento, atinge jovens, adultos e crianças, sendo encarada como uma pandemia e uma condição de vida que afeta o indivíduo em dimensões sociais e psicológicas em todas as classes sociais.

No Brasil, o número de pessoas obesas aumentou 67,8\% entre 2006 e 2018, como aponta uma pesquisa do Ministério da Saúde (2019). E, de acordo com a pesquisa de Vigilância de Fatores de Risco e Proteção para Doenças Crônicas por Inquérito Telefônico - Vigitel (2018), hodiernamente, 55,7\% da população adulta do Brasil está com excesso de peso e 19,8\% está obesa (MINISTÉRIO DA SAÚDE, 2019).

Doenças crônicas relacionadas à dieta, como diabetes, doenças cardiovasculares, hipertensão, acidente vascular cerebral e alguns tipos de câncer, possuem na obesidade e no sobrepeso seu maior fator de risco; sendo a principal consequência do aumento do consumo de alimentos ricos em gorduras e com altos níveis de açúcar em sua composição (BAUMGARTNER et al. 1995). Esses fatores, combinados a uma vida sedentária, aumentam o risco de morte prematura e a condição de adquirir tais doenças, o que prejudica a qualidade de vida do indivíduo (PUSKA; NISHIDA; PORTER, 2003). De acordo com o Instituto Nacional de Câncer (INCA, 2018), a obesidade pode aumentar o risco de incidência de, pelo menos, 13 tipos de cânceres, fato que deve ser encarado não apenas como um problema individual, mas como um problema econômico e de saúde pública.

Martins (2018) considera a obesidade um problema multifatorial, e aponta para a diversidade de esforços em seu enfrentamento, tendo como protagonista o poder público e suas propostas de política pública contra o ambiente obesogênico estimulado pela indústria alimentícia. Desse modo, evidencia-se um desequilíbrio nos sistema de marketing (DOMEGAN et al., 2020), e atores que precisam cumprir com suas funções para minimizar as consequências e apresentar soluções para o problema da obesidade ou sobrepeso. O consumo excessivo de itens nutricionais que causam o aumento na glicemia e o acúmulo de gordura corporal decorrem de mudanças urbanas e comportamentais, nas quais o padrão nutricional se modifica em função de uma ordem econômica, trazendo à sociedade um modelo de consumo alimentar que prioriza a rapidez no preparo, na manutenção e no estoque de alimentos prontos para o consumo (MONTEIRO; LOUZADA, 2015). Tal fato, aliado aos meios de propagação da praticidade alimentar, levam o consumidor a um estado de vulnerabilidade que afeta sua saúde e sua qualidade de vida, dado que a composição desses alimentos se destaca pelo alto teor de gordura e açúcar nem sempre percebidos ou informados.

Dessa forma, interações de mercado e o consumo de produtos ou mensagens de marketing podem induzir o consumidor a momentos de inferioridade no contexto de troca e a situações fora do controle do indivíduo, que o deixam dependente de fatores externos, fixando seu equilibrio no mercado e afetando seu comportamento no cotexto do consumo (BAKER et al., 2005). As condições físicas, motivacionais, sociais e cognitivas que provocam essa vulnerabilidade são ampliadas pelo potencial de dano que o produto causa à saúde física e psíquica do indivíduo (ANDREASEN et al., 1990; HIRSCHMAN, 1992; LUCE; KAHN, 1999; HILL; SHARMA, 2020) e por mensagens publicitárias (MOORE et al., 1996) presentes nos discursos tanto dos veículos de divulgação em massa quanto na própria embalagem do produto.

Disponibilizar essas informações de forma clara se mostra essencial, uma vez que as condições de vida da população, como de trabalho e falta de tempo na configuração da sociedade atual, impulsionam uma alimentação menos saudável dos indivíduos, que consomem mais alimentos industrializados, considerados mais disponíveis ou mais fáceis do que alimentos mais saudáveis e naturais (BALEM, et al., 2017), especialmente quando consideramos o crescente número de pessoas obesas ou com sobrepeso no país, como já anteriormente citado. Nesse sentido, considera-se haver uma intensificação do estado de vulnerabilidade do consumidor em sua alimentação.

À vista disso, é preciso considerar o contexto de crescimento do número de indivíduos obesos ou com sobrepeso em nações industrializadas e naquelas em desenvolvimento (BAKER, et al., 2020), assim como as condições de mercado que, carregadas de falhas ou práticas predatórias (KLEIN, 2019), ocultam ou falseiam informações sobre o teor nutricional dos alimentos. Isto, junto à propagação da agilidade alimentar e das condições urbanas de trabalho, tempo e deslocamento, para entender que há uma intensificação do estado de vulnerabilidade desse grupo quanto ao consumo de alimentos industrializados. 
A vulnerabilidade do consumidor é o público-alvo da Pesquisa Transformativa do Consumidor (TCR), que se concentra nos desequilíbrios das forças dos atores que estão envolvidos no consumo, buscando promover alguma mudança social a partir da mudança no seu comportamento de consumo (ÁSSIMOS et al., 2019).

No campo da pesquisa transformativa, um levantamento realizado por Andrade et al. (2018) revela as temáticas pesquisadas nacionalmente, entre elas: o consumidor com deficiência motora em hipermercados (MANO, 2014); o consumidor deficiente e os serviços de hotelaria (MANO, 2014); mulheres e materialismo (COELHO et al., 2015); consumo de serviços de beleza por pessoas com deficiência visual (BATINGA, CORREA, \& PINTO, 2016); violência financeira ao consumidor idoso de baixa renda (ALMEIDA; BATINGA; ÁSSIMOS \& PINTO, 2017).

No contexto das pesquisas que abordam o estudo da obesidade, tem-se um rompimento dos limites do campo das ciências da saúde, visto que periódicos internacionais, como Journal of the American Dietetic Association, Journal of Public Health, International Journal of Obesity, Journal of Nutrition Education \& Behaviour e Nutrition Review, valemse de conceitos que envolvem a educação alimentar (BURTON et al., 2006) e a prática de exercícios físicos (BOOTH et al., 2001), direcionando seus trabalhos à análise das causas e das consequências desse problema de saúde.

Tal amplitude confirma a multidisciplinaridade das pesquisas científicas na busca de soluções para o problema da obesidade, que possui no seu cerne o comportamento de consumo e seus determinantes. Nesse contexto, estudos no campo do consumo direcionados à obesidade focam principalmente na educação do consumidor (GOLDBERG; GUNASTI, 2007) e na vulnerabilidade da criança no citado contexto (MOORE; RIDEOUT, 2007), em que a finalidade é promover uma cultura de consumo voltada para o bem-estar.

Entender como o sistema de marketing funciona e o papel dos seus agentes nos processos de troca, bem como os desequilíbrios que são encontrados que desestabilizam o sistema (DOMEGAN et al., 2020), causando problemas como a vulnerabilidade do consumidor (BAKER et al., 2005), é importante para que se consiga alcançar o crescimento econômico (LAYTON, 2009), mas para que também se alcance a qualidade de vida e, consequentemente, o bem-estar de grupos vulneráveis, que, no caso da presente pesquisa, trata-se do grupo de pessoas obesas ou com sobrepeso.

A obesidade é, atualmente, um problema global. Os dados são de que $40 \%$ de toda a população mundial está acima do peso - o que significa o triplo de pessoas obesas a mais do que havia 40 anos atrás (Organização Mundial da Saúde - OMS, 2018). No Brasil, um em cada dois adultos e uma em cada três crianças encontram-se nessa condição (Instituto Brasileiro de Geografia e Estatística - IBGE, 2010). Estudo recente publicado pela Cancer Research UK (2018), do Reino Unido, indicou que a geração nascida entre os anos 1980 e 1990 - os chamados millennials - está no caminho de se tornar o grupo com maior incidência de sobrepeso já registrada. Portanto, as mudanças no sistema alimentar global, juntamente com o aumento do comportamento sedentário, parecem ser as principais causas da pandemia de obesidade.

Justifica-se, então, estudar a vulnerabilidade do indivíduo obeso quanto ao consumo de alimentos industrializados à luz da pesquisa transformativa, para que seja possível aproximar-se de uma realidade que propicie ao poder público tomar decisões de saúde pública como um elemento constituinte do sistema de marketing a partir do comportamento de consumo (KLEIN, 2009). Sob essa perspectiva, o presente artigo propõe que os aspectos de malefícios à saúde sejam considerados sob o ponto de vista de desequilíbrio no sistema que envolve o mercado e a sociedade.

A pesquisa transformativa posiciona-se num contexto de fluxo de conhecimento que envolve estudos sobre aprendizagem (JAYANTI; SINGH, 2010), cultura de consumo (OZANNE et al., 2011) e políticas públicas (SCHULTZ, 2012), por exemplo, e vale-se de múltiplas abordagens e paradigmas para seu desenvolvimento. Apropriada disso, propõese a promover mudanças nas práticas de consumo que desequilibram o sistema de trocas, em que se esforça para trazer benefícios à qualidade de vida do consumidor. Logo, encaixa-se aqui, como objeto de estudo, a vulnerabilidade no contexto de troca dos indivíduos obesos ou com sobrepeso, em que, especificamente para este artigo, objetiva-se discutir como se dá a vulnerabilidade dos consumidores obesos ou com sobrepeso a partir do consumo alimentar.

Apresentada a problemática, a estrutura do trabalho conta com as subseções que serão apresentadas posteriormente: (i)Sistemas de Marketing, partindo do ponto de vista do macromarketing, que tem como objeto de estudo as trocas e análises a partir de uma perspectiva holística de sistemas; a (ii)Pesquisa Transformativa do Consumidor, que está no cerne dos estudos de (iii) Vulnerabilidade do Consumidor, causada pelo desequilíbrio dos sistemas de marketing. E como objeto do estudo, na sequência, é explanada a (iv) Obesidade doConsumidor, seguindo para as (v) Considerações Finais, que apresentam as principais conclusões do estudo.

\section{SISTEMAS DE MARKETING}

A visão do marketing como atividade social, irrestrita à atividade comercial, ganhou força quando autores como Kotler e Levy (1969) ampliaram o conceito de marketing, estabelecendo a troca como elemento central e estendendo a prática do marketing para organizações sem fins lucrativos. O conceito de marketing, atribuído pela Associação Americana de Marketing (AMA), sofreu modificações e passou a incluir a sociedade como uma unidade participante do sistema de marketing fundamental para o alcance do seu equilíbrio.

Pautado essencialmente no macromarketing, os sistemas de marketing são uma rede de indivíduos, grupos e/ou entidades ligadas direta ou indiretamente por meio da participação em troca econômica que cria, monta, transforma e 
produz sortimentos de produtos disponíveis de acordo com a demanda dos consumidores (DOMEGAN et al., 2020). Os sistemas de marketing estão relacionados à troca, envolvem a compra, venda, negociação, incluídas nos fluxos de bens, informações, dinheiro e influência. Tais ações ocorrem por meio da interação dos agentes, gerando ofertas e demandas, posses, propriedades transferidas, lucros e desejos alcançados. Assim, a manutenção do equilíbrio do sistema realiza novos ciclos que se expandem e se aprimoram (COSTA, 2015).

Os sistemas de marketing são estabelecidos em uma perspectiva orgânica, incluindo diversos agentes e atores que se adaptam e reagem ao contexto em que estão inseridos (DOMEGAN et al., 2020). O sistema de marketing, portanto, é dirigido tanto por forças de eficiência, evidenciadas pelo mercado (WILKIE \& MOORE, 1999), quanto por forças sociais, que incluem cultura, legislação, códigos de ética e moral e comportamento do consumidor, sendo baseado em processos coordenados e operando por meio de interações.

Inserida num sistema alimentar, a obesidade é um fenótipo de causas variadas, classificada como uma doença crônica não transmissível e definida como o acúmulo excessivo de tecido adiposo pela extensão corporal, em níveis que possam causar danos à saúde do indivíduo (WHO, 1997; FRANCISCHINI et al., 2000; PINHEIRO et al., 2004). O excesso de gordura torna o sujeito predisposto a doenças não transmissíveis, como o diabetes, as doenças cardiovasculares e os cânceres (ALMEIDA LM, et al, 2017). Além destas, destaca-se também os transtornos sociais e psicológicos por preconceito e discriminação.

A obesidade está relacionada, entre outras causas, com a industrialização dos sistemas alimentares, a mudança tecnológica e a globalização, incluindo o crescimento do mercado e as atividades políticas das empresas transnacionais de alimentos e políticas inadequadas para proteger a nutrição nesses novos contextos (BAKER et al., 2020), apontando falhas no mercado (KLEIN, 2019).

Assim, uma das principais premissas dos estudos de macromarketing é a perspectiva de crescimento a partir do equilíbrio entre os agentes e o acesso ao que é ofertado. A má adaptação dos sistemas de marketing aos ambientes em que operam, por razões estruturais ou funcionais, afeta o crescimento, o bem-estar e, consequentemente, a qualidade de vida. Compreender os sistemas de marketing, portanto, é necessário para um engajamento ativo do marketing com contribuições verdadeiramente positivas para a sociedade (SHULTZ, 2007; LAYTON, 2009).

\section{$3 \quad$ PESQUISA TRANSFORMATIVA DO CONSUMIDOR}

Inserida numa perspectiva mais ampla de visualizar o marketing para além dos estudos gerenciais, a Transformative Consumer Research (TCR) originou-se com o objetivo de abordar problemas sociais. E, como propõe a vertente do marketing que trata disso, intenta alcançar a mudança no comportamento do consumidor transformando-o em agente de suas escolhas (MICK et al. 2012). A realização de trabalhos enquadrados por um problema ou oportunidade fundamental engendra esforços para respeitar, defender e melhorar a vida em relação ao repertório de condições, demandas, potencialidades e efeitos do consumo, que estão no cerne dos esforços da TCR (MICK, 2006).

A pesquisa transformativa do consumidor volta-se para questões outrora pouco debatidas por pesquisadores de marketing, enfocando o consumidor e buscando reequilibrar as relações de poder entre o mercado, os consumidores e o governo. Entre os consumidores, estão os que a TCR considera vulneráveis, quais sejam: idosos, crianças, analfabetos, imigrantes, viciados, pessoas com deficiência, pessoas que vivem em situação de extrema pobreza, entre outros, que podem se classificar com menos capacidade de se defender das consequências negativas do mercado de consumo (ÁSSIMOS et al. 2018).

A TCR é uma iniciativa que estuda o papel que o consumo desempenha nos principais problemas sociais da presente época. O objetivo da pesquisa transformativa é realizar pesquisas práticas que possam ser usadas por consumidores e organizações interessadas em melhorar o bem-estar do consumidor e, assim, conseguir fornecer resultados que se aproximem da realidade (OZZANE et al., 2011; BLOCKER, 2013).

A vulnerabilidade do consumidor, bem como as ações que a potencializam, são estudadas pela TCR. Mick et al. (2012) descreveram os seis pontos de compromisso da pesquisa transformativa, quais sejam: promover o bemestar; promover a diversidade de paradigmas, considerando que não existe um paradigma superior ao outro, e sim paradigmas que são adequados a determinados estudos; executar teorias e métodos de estudo que possuam casos já praticados; apresentar contextos socioculturais e situacionais relevantes para um grupo específico de consumidores; estreitar relações entre pesquisadores e consumidores; e propagar os resultados das pesquisas para que seja atendido o objetivo da TCR. 
Figura 1 - As principais características da TCR.

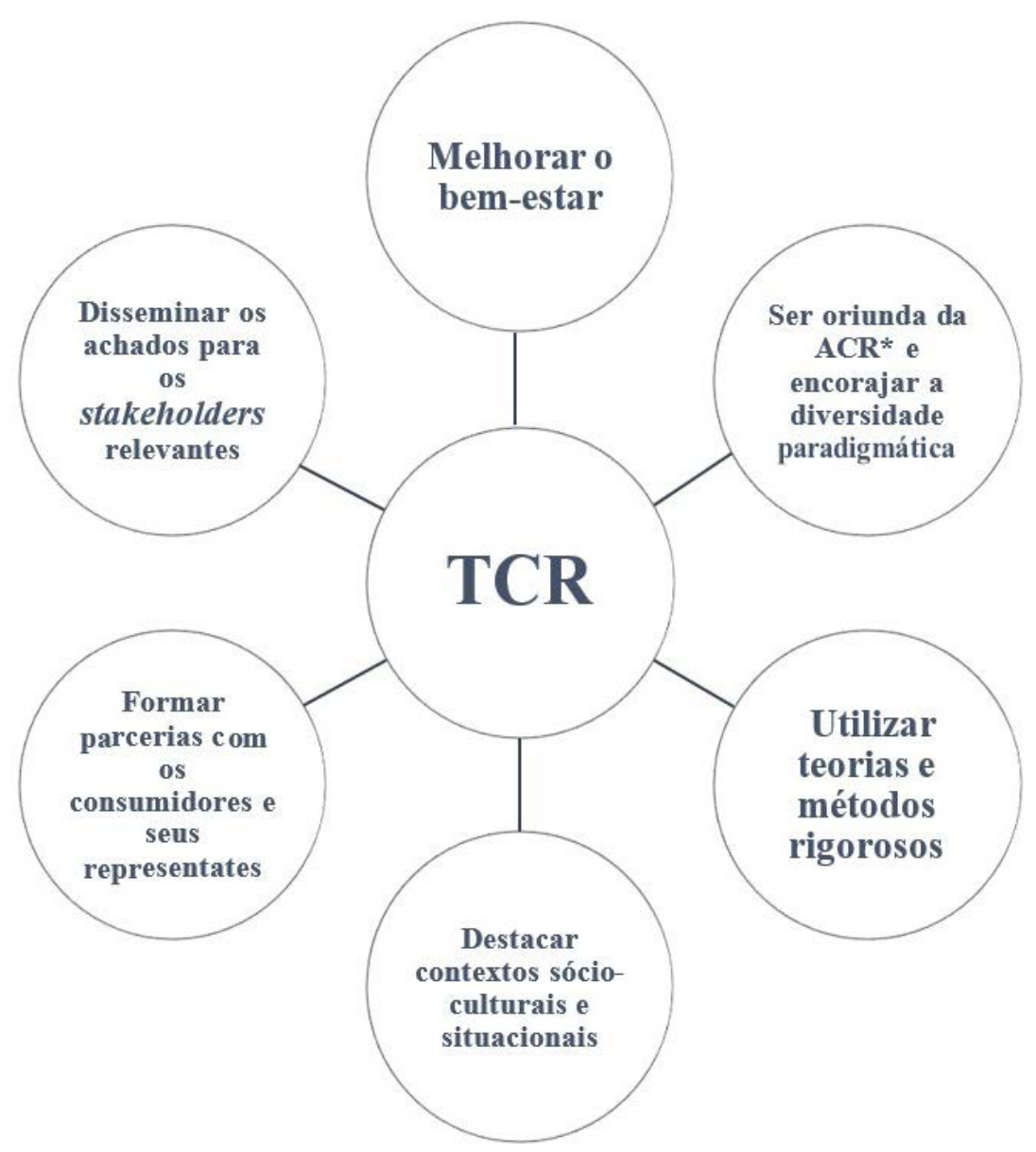

Fonte: Mick et al. (2012, p. 06).

Nota: *ACR - Association of Consumer Research

Ao promover uma agenda de pesquisa, Crocket et al. (2013) dão algumas orientações que diferenciam a pesquisa tradicional da pesquisa transformativa, enfatizando aspectos como o problema de pesquisa e a importância de sua relevância para a promoção do bem-estar social, o incentivo de grupos de pesquisadores interdisciplinares, amostra flexível e criativa embasada cientificamente, coleta de dados inovadora e com participação ativa dos pesquisados, e resultados que sejam traduzidos e disseminados em meios ajustáveis à audiência do grupo que se pretende alcançar.

\section{VULNERABILIDADE DO CONSUMIDOR}

A vulnerabilidade do consumidor é parte significativa nos estudos de marketing, especialmente quando se propõe a estudar o marketing de maneira ampla. Quanto ao consumo, a vulnerabilidade é conceituada como uma situação de incapacidade (que pode ser passageira) que impossibilita a realização dos objetivos de consumo. Portanto, aqui é enxergada como uma condição, pois está relacionada a um estado de exposição a determinados riscos e à capacidade reduzida para se defender ou lidar com implicações negativas.

A definição aqui é proposta em função do contexto de troca, e reforça o que já propõe a TCR ao buscar a melhoria do bem-estar e encorajar a diversidade paradigmática. Ao mesmo tempo que utiliza teorias e métodos rigorosos, ela destaca contextos socioculturais e situacionais ao estudar parcerias com os consumidores e seus representantes e disseminar os achados para os stakeholders relevantes, expondo como desvantagem do consumidor no ambiente de troca as características não controláveis (ANDREASEN; MANING, 1990; SHI, 2017).

Embora os dois estudos afirmem que o conceito de vulnerabilidade do consumidor não está especificamente definido e, ainda assim, fornecerem direcionamentos do que considerar nesse campo, Baker et al. (2005) estabelecem um limite conceitual do que não é vulnerabilidade do consumidor, apontando erros de definição quanto ao contexto de transação. Os autores asseguram que características demográficas, estigmatização, discriminação ou necessidades não satisfeitas provocam uma má interpretação desse conceito, porém reafirmam a falta de consenso na sua definição exata.

Apesar do debate entre os limites do conceito de vulnerabilidade, falta clareza na definição da transitoriedade desse estado, porém os estudos no campo já contribuem para o alcance dessa definição. Reforça-se, assim, a busca 
pelos momentos em que o indivíduo experimenta a vulnerabilidade, o que o leva a tal condição ou se, pelo fato de pertencer a um grupo definido, é sempre vulnerável (BAKER et al., 2005).

Determinados tipos de produtos têm maior probabilidade de causar danos aosconsumidores, especificamente àqueles indivíduos suscetíveis aos mecanismos de indução desse produto. $\mathrm{E}$, para que isso ocorra, há fatores que incrementam essa chance de dano, como as características individuais biofísicas e psicossociais, os estados individuais de tristeza, medo e humor, e as condições externas de discriminação por etnia ou aparência física (MORGAN et al., 1995; BAKER et al., 2005). Existe, também, a questão da compreensão limitada dos rótulos dos produtos, ainda que seja um consenso a necessidade de expor nos alimentos industrializados a tabela nutricional e a lista de ingredientes de maneira clara e objetiva (MARTINS, 2018). Os elementos citados evidenciam que, mesmo diante de circunstâncias informativas, a acessibilidade expressiva e contundente de produtos dessa natureza ampliam a vulnerabilidade do consumidor devido a falta de compreensão dos elementos descritos.

Logo, diante de tais fatores, a proposta desta pesquisa considera a obesidade como um estado real de vulnerabilidade, causado por condições que o levaram a ser e a permanecer doente. Dessa maneira, entender a obesidade como uma doença crônica não transmissível, sob a ótica dos estudos comportamentais, é o cenário que norteia este artigo no sentido de fornecer uma lente interdisciplinar para tratar a questão da obesidade incitando o desenvolvimento de ferramentas que garantam melhoria da qualidade de vida.

\section{A OBESIDADE DO CONSUMIDOR: UMA QUESTÃO INTERDISCIPLINAR}

Consideramos, para fins deste artigo, que uma das externalidades do sistema de marketing em desequilíbrio é a obesidade do consumidor. Este, por sua vez, ocupa uma situação de vulnerabilidade especialmente por se encontrar, a maioria das vezes, em condições assimétricas em relação ao mercado. Por conseguinte, a pesquisa tradicional no marketing não atende com o rigor necessário os consumidores em vulnerabilidade. Nesse ponto, a pesquisa transformativa fornece uma possibilidade de diálogo não apenas com o consumidor obeso, mas com diversas disciplinas que se interessam pelo tema.

Dessa forma, justifica-se analisar o problema da obesidade a partir de uma perspectiva holística, como bem dito no cerne da TCR (MICK, et al., 2012; CROCKET, et al., 2013), e acrescenta-se aqui uma questão interdisciplinar a qual penetra em abordagens das ciências de variadas áreas que se ocupam em prevenir e tratar a obesidade. Assim, entendendo a complexidade da situação de vulnerabilidade envolvendo a obesidade, compreende-se o lugar do marketing e o seu papel nos estudos da área do consumo, principalmente quando o fenômeno pode gerar uma externalidade dessa natureza ao sistema.

A prevenção e os tratamentos da obesidade frequentemente falham em longo prazo - como intervenções comportamentais com o objetivo de reduzir a ingestão de calorias e aumentar o gasto energético -, ou não estão disponíveis, ou são pouco adequados (cirurgia bariátrica) para a maioria das pessoas afetadas. Embora a prevalência da obesidade tenha aumentado em todos os países do mundo, existem diferenças regionais na prevalência e nas tendências da obesidade. Assim, compreender os impulsionadores dessas diferenças regionais pode ajudar a fornecer orientação para as estratégias de intervenção mais promissoras (ALMEIDA, et al., 2017; BLÜHER, 2019).

Tratar a obesidade como doença é ligar-se à última ponta de um sistema complexo que envolve a participação dinâmica de diversos participantes em trocas constantes. Todavia, enquanto profissionais da saúde se ocupam em tratar os efeitos dessas trocas em desequilíbrio, torna-se oportuno que acadêmicos do campo do marketing se dediquem a compreender a lógica por trás dos interesses de quem participa dessas trocas e como elas podem atenuar problemas que levam o consumidor a tal estado de vulnerabilidade.

Considerando a obesidade como um problema pandêmico que precisa ser tratado por diferentes esferas de organizações e sociedade, o principal desafio é traduzir nosso conhecimento das principais causas do aumento da prevalência da obesidade em ações eficazes. Tais ações, portanto, podem incluir mudanças de política que facilitem as escolhas individuais de alimentos com teor reduzido de gordura, açúcar e sal. (SWINBURN et al. 2011; YANOVSK, 2018; DE LORENZO et al., 2020).

Um fato considerado neste artigo como um dos condicionantes a serem investigados na vulnerabilidade é o desconhecimento do valor nutricional. Pode ser citado, por exemplo, a substituição do nome açúcar por carboidratos, realizado pela indústria alimentícia e de bebidas no rótulo nutricional dos produtos, ou ainda a divulgação dos níveis de sódio e gorduras trans dos alimentos, que fazem pouco sentido para quem não discerne os riscos dos termos a sua saúde. Aqui, as áreas de nutrição e comunicação social, com foco na publicidade, dão suporte à criação de embalagens que transpareçam corretamente o valor nutricional dos alimentos e contribuam para a educação do consumidor.

O desequilíbrio causado pela assimetria entre quem oferta o produto e quem consome pode ensejar gatilhos que levam não só à obesidade, como a diversos problemas de saúde. Tais fatores conjugados formam um problema econômico, pois seus custos consideram a diminuição na qualidade de vida, a queda na produtividade do trabalhador, o aumento do número de mortes e a aposentadoria precoce.

Tratando-se da obesidade, destaca-se que os gastos realizados com serviços de saúde e medicação pelos indivíduos obesos chegam a ser o triplo do gasto de um indivíduo com peso normal (FINKELSTEIN et al., 2009). É 
um problema que se soma ao preconceito social e à hostilidade e discriminação contra a obesidade, atribuindo ao indivíduo um estigma de socialmente inadequado, vivenciando situações de rejeição e preocupação exagerada com a imagem, o que reforça a condição de vulnerável.

Essa condição é aqui considerada como contribuinte para o estresse emocional vivido pelo indivíduo obeso, com base no modelo psicossomático da obesidade, que aponta o mecanismo compensatório do consumo de alimentos como fuga emocional. Assim, os alimentos são consumidos também para a diminuição de sensações como ansiedade, depressão, raiva e tristeza (FAITH; GOLIEBTER, 1997).

Consequentemente, a vulnerabilidade do indivíduo obeso, ligada diretamente ao seu comportamento de consumo, além de ser influenciada pela condição social de preconceito e rejeição, é intensificada por construtos latentes surgidos de desordens psiquiátricas e angústias psicológicas, como medo e depressão (TELCH; AGRAS, 1994). Tais condições são agravadas pelo consumo de alimentos industrializados, que funcionam com efeitos viciantes ao considerar que as condições biopsicossociais são retroalimentadas em ciclos de comportamentos repetitivos. Pessoas obesas não conseguem se sentar de forma confortável em assentos comuns, não conseguem passar na catraca de um ônibus, também são tacitamente excluídas de profissões que requerem um padrão que transmita saúde e autocuidado.

Discussões sobre o marketing e a diversidade já denunciavam a insuficiência de produtos e serviços para esse público (CAVALCANTI et al., 2016). Dessa forma, a interdisciplinaridade torna-se ainda mais importante ao considerar que, além do esforço para o equilíbrio das trocas, é necessário não negar que pessoas obesas existem e que precisam de políticas capazes de incluí-las na sociedade e com o bem-estar devido a qualquer outro grupo. A oferta adequada de bens e serviços para alcançar justiça no ambiente de consumo envolve diversas outras disciplinas, como as áreas de turismo, arquitetura, construção civil, design de produtos, gestão de pessoas, assistência social, psicologia, antropologia, entre outras.

\section{CONSIDERAÇÕES FINAIS}

O marketing, durante sua evolução, arraigou-se a uma perspectiva econômica e que, por muito tempo, esteve mais próxima a atender desejos individuais do que sociais (LAYTON, 2007). Entretanto os esforços para tornar o marketing além de uma disciplina provocam uma evolução nos conceitos e a compreensão de que a função do marketing não se limita a criar ofertas que tenham valor para clientes, mas sim valor para sociedade (AMA, 2013).

Por meio da Pesquisa Transformativa do Consumidor, busca-se equilibrar as trocas sob pontos de vista físico, psicológico, econômico e social. Nesse contexto, destaca-se que a alimentação representa também um ato social, e o estreitamento entre o consumo alimentar e saúde é interesse de pesquisa do marketing, uma vez que o desequilíbrio na oferta e consumo tem potencial de gerar danos à população.

O marketing, de um ponto de vista micro, que, segundo Chaganti e Heed (1981), se refere às atividades de marketing em nível individual de empresas, consumidores ou famílias, responsabiliza o consumidor por suas escolhas. Em uma visão ampliada, busca-se em outras disciplinas suporte para entender que nem sempre o consumidor tem clareza sobre suas próprias escolhas. No caso do consumo alimentar, os consumidores podem ser considerados vulneráveis, tanto pela escassez de informações, em níveis adequados disponíveis nos ingredientes, como pela substituição dos termos populares por termos mais científicos.

De algum modo, o marketing torna-se responsável por essa vulnerabilidade dado o alcance social da disciplina. Nesse sentido, propõe-se uma agenda interdisciplinar para atender à complexidade do consumidor e de suas escolhas alimentares, pois, nesse sentido, aliam-se aos estudos de consumo, pesquisas no campo da saúde pública, da nutrição e da economia (BENSON et al., 2018; THIELE; ROSE, 2018). Muitas ações midiáticas alcançam a vulnerabilidade do consumidor à medida que ofertam bem-estar e felicidade nos alimentos e omitem informações importantes sobre o consumo.

Aqui, lança-se sobre a obesidade uma visão interdisciplinar, pois ela é um problema econômico real e de uma complexidade que exige esforços de diferentes áreas para o seu enfrentamento. Muitos consumidores, ainda que não sejam obesos, já estão em condição nutricional que tende ao estado de sobrepeso, enquanto outros apresentam mudança dessa condição por meio de dietas, tratamentos convencionais ou alternativos, e até intervenções cirúrgicas (que já se apresentam como decisões e escolhas de consumo).

É por meio de uma visão interdisciplinar que se assume a existência e a complexidade do problema, além de lançar propriedade aos estudos de consumo acerca da má alimentação, do excesso de produtos industrializados, do sedentarismo e outros comportamentos considerados de risco para o obeso, como alcoolismo e o tabagismo. A TCR é uma via para compreender a condição de vulnerabilidade dos consumidores obesos, e, por sua essência, é interdisciplinar, ao passo que a integração sistêmica dos diversos participantes nas trocas é fundamental para compreender o papel de cada ator na dinâmica do consumo.

Como debatido, a interdisciplinaridade atua num eixo que envolve abordagens capazes de, por exemplo, contribuir para eliminar o paradoxo no qual o mercado, que estimula o consumo de alimentos ricos em substâncias que, em excesso, se tornam deletérias, dita um padrão de corpo saudável e estetiza o modelo de corpo perfeito,

Há que se olhar para o consumidor buscando entender como é possível colaborar para atenuar sua condição vulnerável, pois os consumidores sofrem pelos males da obesidade, apresentam comorbidades emocionais por 
vezes acompanhadas de baixa autoestima atribuídas à autoimagem. Por outro lado, os consumidores que já estão na condição de obesos ocupam o eixo interdisciplinar à medida que necessitam de políticas e cuidados específicos para se sentirem parte da sociedade.

Desse modo, o debate precisa transcender aacademia, chegando à sociedade e ao mercado. Hoje, por exemplo, há múltiplas opções rotuladas como alimentos saudáveis e, através dos veículos de comunicação, constantemente há divulgação de dietas da moda. Com isso, o mercado enxerga uma possibilidade de atuação investindo em franquias de fast foods adaptadas a um estilo saudável, shakes para substituir refeições tradicionais e produtos "zero". Tais produtos geralmente apresentam preço superior ao produto convencional, mas nem sempre cumprem o que prometem, e são nessas esferas de atuação que se implica os desafios do marketing.

O preconceito com pessoas obesas tem afastado esse público do acesso ao mercado de trabalho e das promoções em seus cargos. É reconhecido pela Organização Mundial do Trabalho que a discriminação baseada no peso impõe barreiras à realização de um trabalho digno e é empecilho passível de intervenção (OIT, 2011). Araújo et al. (2018) citam que, em 2011, foi realizado um concurso público para professores, mas que alguns tiveram problemas em suas nomeações por justificativa médica que ratificava a "obesidade como doença incapacitante para o trabalho, principal motivo de reprovação do concursado visto como legítimo pela perícia médica" (ARAUJO et al., 2018, p.75).

Nesse sentido, os autores supracitados destacam um ponto relevante ao concluir que a sociedade, que cria os obesos, não os abriga. Diante de tantos apelos por uma abordagem interdisciplinar, é necessário que o marketing reconheça sua responsabilidade também na intervenção contra a obesidade. A partir da perspectiva transformativa do consumidor, pode-se ter uma visão ampla de todos os fatores que impactam a vida das pessoas com obesidade. Deve-se, assim, avaliar a participação em conjunto de pesquisas em diversas áreas, a fim de que o debate sobre esse tema extrapole os limites acadêmicos e chegue ao mercado, revertendo-se em melhores alternativas aos consumidores e promovendo a inclusão.

Este artigo busca contribuir para a discussão sobre o comportamento de consumo que tem a obesidade como uma consequência, no qual o estado de sobrepeso provoca danos físicos e psicológicos, como cânceres e depressão, respectivamente. Diante de um fenômeno de consumo que pode ser observado tanto pela lente econômica quanto pela de saúde, o poder público pode atuar por meio de uma combinação entre campanhas de educação alimentar e medidas regulatórias sobre alimentos ultraprocessados, por exemplo, pois há estudos que apontam uma eficácia de um imposto que afeta o preço do produto e promove alterações no consumo (BOGART, 2013).

Há de se contestar, porém, até que ponto um preço se ajusta em resposta ao imposto dado que o comportamento do consumidor é mutável. Poderia o consumidor tanto mudar para uma alternativa mais saudável quanto para uma opção mais acessível, mesmo que prejudicial. Aqui, caberiam estudos no campo do marketing sobre sensibilidade aos preços de produtos ultraprocessados ou estudos no campo da economia sobre essa possível taxação e as externalidades que envolvem a obesidade, como aumento dos gastos com a saúde pública. Ainda aqui, estudos no campo da comunicação sobre mensagens publicitárias de produtos saudáveis e sua eficácia sobre a mudança de camportamento, experimentos, podem auxiliar nesse intuito.

Portanto, a amplitude das alterações no consumo, como uma mudança de comportamento, são bastante variadas e podem ser substanciais em alguns casos, como no caso do imposto francês sobre o refrigerante (ALLAIS; BERTAIL; NICHĖLE, 2010). Além disso, estudos, em particular aqueles baseados em simulações de demanda, mostram que o imposto é regressivo por natureza e tem impactos maiores nas famílias de baixa renda. Assim, merecem atenção as externalidades não intencionais sobre os nutrientes não alcançados pelo imposto, como as vitaminas.

Nesse sentido, desde o consumo em si e seus contextos de influência nas escolhas e nas decisões até a taxação de alimentos, permeando aspectos psicológicos, educacionais e de saúde pública, a compreensão da obesidade como um fenômeno de consumo - logo, uma externalidade econômica - tem suas proposta de discussão e solução na interdisplinaridade. Assim, é um debate característico da pesquisa transformativa, pois busca uma mudança de comportamento, e não se alcança tal mudança sem que se tenha à frente um espectro de conhecimentos contribuindo entre si.

\title{
REFERÊNCIAS
}

ADREASEN, A. R.; MANNING, J. The dissatisfaction and complaining behavior of vulnerable consumers. Journal of Consumer Satisfaction/Dissatisfaction and Com- plaining Behavior, [S. I.], v. 3, p. 12-20, 1990.

ALLAIS, O.; BERTAIL, P.; NICHĖLE, V. The effects of a fat tax on french households' purchases: a nutritional approach. American Journal of Agricultural Economics, [S. I.], v. 92, n. 1, p. 228-245, 2010.

\author{
ALMEIDA, G. T.; ÁSSIMOS, B. M.; BATING, G. L.; PINTO, M. R. A abordagem
}

transformative consumer research (tcr): apontamentos paradigmáticos, discussões metodológicas e evolução das investigações internacionais e nacionais. In: ENCONTRO DAANPAD - ENANPAD, 42, 2018, Curitiba. Anais [...]. Curitiba: Associação 
Nacional de Pós- graduação e Pesquisa em Aministração, 2018.

ALMEIDA, L. M. et al. Estratégias e desafios da gestão da atenção primária à saúde no controle e prevenção da obesidade, Revista Eletrônica Gestão \& Saúde, Brasília, v. n. 1, p. 114-139, 2017.

ANDRADE, J. H. F. Regional policy approaches and harmonization: a latin american perspective. International Journal of Refugee Law, Oxford, v. 10, n. 3, p. 389-409, 1998, p. 400.

ARAÚJO, L. S. et al. Preconceito frente à obesidade: representações sociais veiculadas pela mídia impressa. Arquivos Brasileiros de Psicologia, [S, I.], v. 70, n. 1, p. 69- 85, 2018.

ÁSSIMOS, B. M.; ALMEIDA, G. T.; BATINGA, G. L.; PINTO, M. R. A construção de tecnologias sociais como produto das Pesquisas Transformativas do Consumidor: uma inspiração psicossociológica. Revista Eletrônica Gestão \& Sociedade, [S. I.], v. 13, n. 3, p. 2710-2735, 2019.

ASSOCIATION FOR CONSUMER RESEARCH. Transformative consumer research: inspiring scholarship for collective and personal well-being. Disponível em: Link. Acesso em: 21 out. 2018.

BALEM, T. A; ALVES, E. O.; COELHO, J. C.; MELLO, A. L. P. As transformações alimentares na sociedade moderna: a colonização do alimento natural pelo alimento industrial. Revista Espacios, [S. I.], v. 38, n. 47, 2017.

BAKER, S. M.; GENTRY, J. W.; RITTENBURG, T. L. Building understanding of the domain of consumer vulnerability. Journal of Macromarketing, v. 25, n. 2, p. 128-139, dez. 2005.

BATINGA, G. L.; CORREA, R. S.; PINTO, M. R. O consumo de serviços de beleza por mulheres deficientes visuais na perspectiva da transformative consumer research. In: EMA, 7., Belo Horizonte. Anais [...]. Belo Horizonte: ANPAD, 2016.

BAUMGARTNER, R. N.; HEYMSFIELD, S.B.; ROCHE, A. F. Human body composition and the epidemiology of chronic disease. Obesity Res., [S. I.], v. 3, p. 73-95, 1995.

BENSON, Tony et al. The impact of nutrition and health claims on consumer perceptions and portion size selection: results from a nationally representative survey. Nutrients, v. 10, n. 5, p. 656, 2018.

BLOCKER, C. P. et al. Understanding poverty and promoting poverty alleviation through transformative consumer research. Journal of Business Research, [S. I.], v. 66, n. 8, p. 1195- 1202, 2013.

BOGART, William A. Regulating obesity?: government, society, and questions of health. Reino Unido: Oxford University Press, 2013.

BOOTH, S. L. et al. Environmental and societal factors affect food choice and physical activity: rationale, influences, and leverage points. Nutrition Reviews, [S. I.], v. 59, n. 3, p. S21-S36, 2001.

BRASIL. Ministério da Saúde. Secretaria de Vigilância em Saúde. Departamento de Vigilância de Doenças e Agravos não Transmissíveis e Promoção da Saúde.Vigitel Brasil 2016: vigilância de fatores de risco eproteção para doenças crônicas por inquérito telefônico. Brasília: Ministério da Saúde, 2017. 160f.

BURTON, S. et al. Attacking the obesity epidemic: the potential health benefits of providing nutrition information in restaurants. Journal Information, [S. I.], v. 96, n. 9, 2006.

CAVALCANTI, H. T. et al. Marketing e diversidade: prazer, eu existo. Diálogo, [S. I.], n. 32, p. 203-224, 2016.

COSTA, F. J. Marketing \& Sociedade. João Pessoa: Editora UFPB, 2015.

CROCKETT, D. et al. Conceptualizing a transformative research agenda. Journal of Business Research, [S. I.], v. 66, n, 8, p. 1171-1178, 2013.

DOMEGAN, C. et al. Marketing systems: a listen, learn, leverage framework. Journal of Macromarketing, [S. I.], v. 32, n. 11-12, p. 1123-1144, 2020. 
FAITH, M. S; ALLISON, D. B; GELIEBTER, A. Emotional eating and obesity: theoretical considerations and practical recommendations. In: DALTON S. Overweigth and weight management. Maryland: Aspen Publishers, 1997. p. 439-65.

FINKELSTEIN, E. A. et al.Annual medical spending attributable to obesity: payer-and service-specific estimates. Health Aff [S. I.], v. 28 n. 5, p. 822-831, 2009.

FRANCISCHI, R. P. et al. Obesidade: atualização sobre sua etiologia, morbidade e tratamento. Rev. Nutr, [S. I.], v. 13, n. 1, p. 17-28, 2010.

GOLDBERG, M. E.; GUNASTI, K. Creating an environment in which youths are encouraged to eat a healthier diet. Journal of Public Policy \& Marketing, [S. I.], v. 26, n. 2, p. 162-181, 2007.

HIRSCHMAN, E. C. The consciousness of addiction: toward a general theory of compulsive consumption. Journal of Consumer Research, [S. I.] v. 19, p. 155-79, 1992.

INSTITUTO BRASILEIRO DE GEOGRAFIA E ESTATÍSTICA. Pesquisa nacional de saúde: 2013: ciclos de vida: Brasil e grandes regiões. Rio de Janeiro: IBGE, 2015.

JAYANTI, R. K.; SINGH, J. Pragmatic learning theory: distributive consumer learning in online communities. Journal of Consumer Research, [S. I.], v. 36, p. 1058-1081, 2010.

KOTLER, P. LEVY, S. Broadening the concept of marketing. Journal of Marketing, [S. I.], v. 33, p. 10-15, 1969.

LAYTON, R. A. Marketing systems-a core macromarketing concept. Journal of Macromarketing, [S. I.], v. 27 , n. 3, p. 227-242, 2007.

LAYTON, R. A. On economic growth, marketing systems, and the quality of life. Journal of Macromarketing, [S. I.], v. 29, n. 4, p. 349-362, 2009.

LOUZADA, M. L. C. et al. Alimentos ultraprocessados e perfil nutricional da dieta no Brasil. Revista de Saúde Pública, [S. I.], v. 49, p. 38, 2015.

LUCE, M. F.; KAHN B. E. Avoidance of vigilance? The psychology of false-positive test results. Journal of Consumer Research, [S. I.], v. 26, p. 242-59, 1999.

MANO, R. F. Consumidor com deficiência: implicações de fatores pessoais e contextuais no consumo varejista de João Pessoa-PB. 2014. Dissertação (Mestrado em Adminitração) - Universidade Federal da Paraíba, João Pessoa 2014.

MARTINS, A. P. B. É preciso tratar a obesidade como um problema de saúde pública. Revista de Administração de Empresas - RAE, São Paulo, v. 58, n. 3, p. 337-341, 2018.

MICK, D. G. et al. (ed.). Transformative consumer research for personal and collective well-being. New York: Routledge, 2012.

MICK, D. G. Meaning and mattering through transformative consumer research. In: PECHMANN, Cornelia; PRICE, Linda L. (ed.) Advances in consumer research, v. 33, Duluth: Association for Consumer Research, p. 1-4, 2006.

MOORE, D. J; JEROME D. W.; WILLIAM J. Q. Target marketing of tobacco and alcohol related products to ethnic minority groups in the U.S. Journal of Ethnicity and Disease, [S. I.], v. 6, p. 83-98, 1996.

MOORE, E. S.; RIDEOUT, V. J. The online marketing of food to children: is it just fun and games? Journal of Public Policy \& Marketing, [S. I.], v. 26, n. 2, p. 202-220, 2007.

MORGAN, F. W.; SCHULER D. K.; STOLTMAN J. J. A framework for examining the legal status of vulnerable consumers. Journal of Public Policy and Marketing, [S. I.], v. 14 n. 2, p. 267-77, 1995.

OLIVEIRA, Michele Lessa de. Estimativa dos custos da obesidade para o Sistema Único de Saúde do Brasil. 2013. 95f. Tese (Doutorado em Nutrição Humana) - Universidade de Brasília, Brasília, 2013. 
ORGANIZAÇÃO MUNDIAL DA SAÚDE. Obesity and overweight fact sheet. Disponível em: http://www.who.int/ mediacentre/factsheets/fs311/en/. Acesso em: 23 out. 2018.

ORGANIZAÇÃO INTERNACIONALDO TRABALHO. Igualdade no trabalho: um desafio contínuo. Relatório Global no quadro do seguimento da Declaração sobre os Princípios e Direitos Fundamentais no Trabalho. Genebra: OIT, 2011.

PINHEIRO, Anelise Rizzolo de Oliveira; FREITAS, Sérgio Fernando Torres de; CORSO, Arlete Catarina Tittoni. Uma abordagem epidemiológica da obesidade. Rev. Nutr., Campinas, v. 17, n. 4, p. 523-533, out./dez., 2004.

PUSKA, P.; NISHIDA, C.; PORTER, D. Global estrategy on diet, physical activity and health. [S. I.]: World Health Organization, 2003.

RAJAN, T. M.; MENON, V. Psychiatric disorders and obesity: a review of association studies. Journal of Postgraduate Medicine, [S. I.], v. 63, n. 3, p. 182, 2017.

$\mathrm{SHI}, \mathrm{H}$. Y. et al. The concept of consumer vulnerability: scale development and validation. International Journal of Consumer Studies, v. 41, n. 6, p. 769-777, 2017.

SHULTZ, C. J. et al. Marketing and public policy: transformative research in developing markets. Journal of Public Policy \& Marketing, [S. I.], v. 31, n. 2, p. 178-184, 2012.

SHULTZ, C. J. Marketing as constructive engagement. Journal of Public Policy \& Marketing, [S. I.], n. 26, p. 293301, 2007.

SOUZA, J. H. J.; MELO, F.V. S. Moda "Só para maiores": experiência de consumo de pessoas obesas em lojas especializadas de vestuário plus size. Revista Administração em Diálogo, [S. I.] , v. 20, n. 3, p. 110-123, 2018.

SPIEKER, E. A.; PYZOCHA, Natasha. Economic impact of obesity. Primary Care: Clinics in Office Practice, [S. I.], v. 43, n. 1, p. 83-95, 2016.

STEWART, C. R.; YAP, S. Low literacy, policy and consumer vulnerability: are we really doing enough? International Journal of Consumer Studies, [S. I.], v. 44, n. 4, p. 343-352, 2020.

TELCH C. F; AGRAS W. S. Obesity, binge eating and psychopathology: are they related? Int J Eat Disord., [S. I.], v. 15, n. 1, p. 53-61, 1994.

THIELE, S.; ROOSEN, J. Obesity, fat taxes and their effects on consumers. Regulating and managing food safety in the EU. [S. I.]: Springer, 2018. p. 169-193.

WHO. Obesity: preventing and managing the global epidemic. Report of WHO consultation on obesity. Geneva: World Health Organization, 1997.

WILKIE, W. L.; MOORE, E. S. Marketing's contributions to society. The Journal of Marketing, [S. I. ]. v. 1, p.198218, 1999. 


\section{Contato:}

Bruna Lourena de Lima Dantas

E-mail: brunalimadantas.adm@gmail.com

Thiago Assunção de Moraes

E-mail: thiagoassuncao@pcs.uespi.br

Hellen Taynan da Silva Cavalcanti

E-mail: hellentaynan@gmail.com

Janayna Souto Leal

E-mail: leal.janayna@gmail.com

Bianca Gabriely Ferreira Silva

E-mail: biianca_ferreira@hotmail.com

Submetido em: 31/08/2019

Revisado em: $15 / 10 / 2020$

Aprovado em: 07/12/2020 